\title{
Manifestations of Pregabalin Withdrawal
}

\author{
Alexandra Hamlyn*, Kalvin Foo, Anum Bhatia and Bradford Bobrin \\ Cooper University Hospital, Camden, New Jersey, United States
}

\begin{abstract}
Pregabalin is an analog to isobutyl $\gamma$-aminobutyric acid (GABA), and is commonly utilized as an anticonvulsant and medication for neuropathic pain. Pregabalin withdrawal has not been well documented, but is commonly described as a constellation of symptoms including anxiety, tachycardia, diaphoresis, nausea, and aggression. We report a case of pregabalin withdrawal with a primary presentation of akathisia, which has yet to be described in current literature.
\end{abstract}

Keywords: Pregabalin; Akathisia; Nausea; Anxiety; Pain

\section{Introduction}

Pregabalin is a commonly used anticonvulsant and neuropathic pain medication. It is an analog to isobutyl $\gamma$-aminobutyric acid (GABA), the major inhibitory neurotransmitter in the body, but does not bind to GABA receptors [1]. Pregabalin is structurally similar to gabapentin and works by binding the alpha-2 subunits on calcium channels and therefor reducing calcium influx, which reduces the release of norepinephrine and glutamate. Furthermore, animal studies have shown that pregabalin most likely exhibits its antiepileptic and decrease of neuropathic pain by interfering with the release of substance $\mathrm{P}$ and calcitonin gene-related peptide [1]. The advantage of pregabalin over gabapentin a more consistent, $>90 \%$ bioavailability compared to gabapentin's inversely proportional bioavailability to dosing $[2,3]$. Both agents, gabapentin and pregabalin, tend to have similar withdrawal side effects, and are both associated with anxiety, tachycardia, diaphoresis, and aggression [4,5]. Gabapentin has also been associated with akathisia; however, this had not yet been described with pregabalin withdrawal [6].

\section{Case Presentation}

A 53 year old male with a history of alcohol use disorder, bipolar disorder and peripheral neuropathy presented to the ER by himself reporting that he had finished a 7 day drinking binge. The patient's home medication regiment consisted of quetiapine, pregabalin $150 \mathrm{mg}$ TID, and citalopram. It is unclear how long the patient had not been taking his pregabalin as he reported taking it sporadically during his 7 days of drinking; however, he had been compliant with medications before that period.

At the time of presentation, he appeared tremulous, diaphoretic, and was grinding his jaw throughout the presentation; however, all vitals were stable. The patient reported significant anxiety, and his CIWA was then measured to be 9 with high risk for progression. $\mathrm{He}$ was started on alcohol withdrawal precautions with diazepam $5 \mathrm{mg}$. In the meantime, his home medications were stopped.

The next day, his CIWA increased to 19, with increased jaw movements, tremor, and anxiety in the setting of IV lorazepam $2 \mathrm{mg}$ Q6 PRN administration. His symptoms persisted with the addition of baclofen on day 3 of his admission. On day 4 of his stay, his CIWA scale had still not decreased, and at this time, his pregabalin $150 \mathrm{mg}$ TID was restarted. One day after the Pregabalin was restarted; his tongue movement and tremor began to diminish. He had full resolution of symptoms by day 7 , which was 3 days after the restart of pregabalin. The patient was safely discharged on day 7 .

\section{Discussion}

Few descriptions of pregabalin withdrawal have been documented as per our literature review. Literature documenting pregabalin withdrawal describes symptoms of nausea, diarrhea, headache, spasticity, and even hallucinations, but there are no reports of pregabalin causing akathisia [1].

GABA is linked to multiple withdrawal syndromes through the benzodiazepine drug class, alcohol, and gabapentin [6]. While benzodiazepines bind specifically to GABA neurons and gabapentin binds nonspecifically in the central nervous system, pregabalin's structural similarity and effects are reasonably linked to GABA mediated withdrawal.

Akathisia is often reported as a drug induced effect, but seldom as an effect of withdrawal. Drug-withdrawal akathisia has been reported as far back as 1960 with phenothiazine, an early psychotropic medication [7]. However, while literature review suggests that it can be reasonably caused by neuroleptics, prevalence and risk factors are not known [7].

It is unlikely that this patient was suffering from alcohol withdrawal for multiple reasons. It is important to note that two to six hours after a person has ceased alcohol consumption, he/she will start to exhibit signs of withdrawal such as nausea, vomiting, diaphoresis, tremulousness, as well as tachycardia, hypertension and tachypnea [8]. These symptoms can be aborted by the administration of IV benzodiazepine, which will start to take affect within hours from the first dose [8]. This patient was given diazepam on day 1 , which had little to no effect on his symptoms. This patient's symptoms persisted until day 4 and only subsided when pregabalin was given. Additionally, when this patient presented to the $\mathrm{ER}$, the patient did not have hypertension, tachypnea, or tachycardia that you would expect to see in alcohol withdrawal. This patient also did not progress to severe stages of alcohol withdrawal such as hallucinations, disorientation, psychosis, or seizures, which you would expect to, see 7 to 48 hours after the patient's last drink [8].

\section{Conclusion}

Pregabalin is a powerful agent that is tolerated well for multiple

*Corresponding author: Alexandra Hamlyn, Cooper University Hospital, Camden, New Jersey, United States, Tel: +1 856-342-2000; E-mail: ahamlyn08@gmail.com

Received: August 27, 2017; Accepted: September 19, 2017; Published: September 26, 2017

Citation: Hamlyn A, Foo K, Bhatia A, Bobrin B (2017) Manifestations of Pregabalin Withdrawal. J Psychiatry 20: 418. doi:10.4172/2378-5756.1000418

Copyright: @ 2017 Hamlyn A, et al. This is an open-access article distributed under the terms of the Creative Commons Attribution License, which permits unrestricted use, distribution, and reproduction in any medium, provided the original author and source are credited 
indications [9]. While prescription practices of the medication dictate that discontinuation of the medication should include a taper, it is important that this additional side effect is documented in the event of sudden discontinuation.

\section{References}

1. Shneker BF, McAuley JW (2005) Pregabalin: A new neuromodulator with broad therapeutic indications. Ann Pharmacother 39: 2029-037.

2. Corrigan BW, Pool WF, Posvar EL, Strand JC, Alvey CW, et al. (2001) Metabolic disposition of pregabalin in healthy volunteers (abstract). Clin Pharmacol Ther 69: 18

3. Elwes RD, Binnie CD (1996) Clinical pharmacokinetics of newer antiepileptic drugs. Lamotrigine, vigabatrin, gabapentin and oxcarbazepine. Clin Pharmacokinet 30: 403-415.
4. Braid JJ, Kirker SG, Baguley IJ (2012) Spasticity increases during pregabalin withdrawal. Brain Inj 27: 120-124.

5. Norton JW (2001) Gabapentin withdrawal syndrome. Clin Neuropharmacol 24: 245-246.

6. See S, Hendriks E, Hsiung L (2011) Akathisia induced by gabapentin withdrawal. Ann Pharmacother 45: e31.

7. Sachdev P (1995) The epidemiology of drug-induced akathisia: part II. Chronic tardive, and withdrawal akathisias. Schizophr Bull 21: 451-461.

8. Stehman CR, Mycyk MB (2013) A rational approach to the treatment of alcohol withdrawal in the ED. Am J Emerg Med 31: 734-742.

9. Zhang SS, Wu Z, Zhang LC, Zhang Z, Chen RP, et al. (2014) Efficacy and safety of pregabalin for treating painful diabetic peripheral neuropathy: A metaanalysis Acta Anaesthesiol Scand 59: 147-159. 